\title{
Image Registration in Multi-Modal Scanning Microscopy: A Solar Cell Case Study
}

\author{
Niklas Pyrlik \\ Deutsches Elektronen-Synchrotron \\ Universität Hamburg \\ Hamburg, Germany \\ Giovanni Fevola \\ Deutsches Elektronen-Synchrotron \\ Hamburg, Germany \\ Andreas Schropp \\ Deutsches Elektronen-Synchrotron \\ Hamburg, Germany
}

\author{
Christina Ossig \\ Deutsches Elektronen-Synchrotron \\ Universität Hamburg \\ Hamburg, Germany \\ Jan Garrevoet \\ Deutsches Elektronen-Synchrotron \\ Hamburg, Germany \\ Michael E. Stuckelberger* \\ Deutsches Elektronen-Synchrotron \\ Hamburg, Germany \\ *michael.stueckelberger@ desy.de
}

\author{
Catharina Ziska \\ Deutsches Elektronen-Synchrotron \\ Universität Hamburg \\ Hamburg, Germany \\ Gerald Falkenberg \\ Deutsches Elektronen-Synchrotron \\ Hamburg, Germany
}

\begin{abstract}
Scanning probe measurements are an indispensable tool of solar cell research today, and the compatibility with simultaneous acquisition of complementary measurement modes is a particular strength. However, multi-modal data acquisition is often limited by different scan-parameter requirements. As a consequence, the modalities may be assessed subsequently rather than simultaneously. In this instance, image registration serves as a tool to align two-dimensional datasets at nanoscale. Here, we showcase an example of two subsequent scanning $X$ ray microscopy measurements of solar cells with a $\mathrm{Cu}(\mathrm{In}, \mathrm{Ga}) \mathrm{Se}_{2}$ absorber, the first measurement being optimized for $\mathrm{X}$-ray beam induced current and the second for X-ray fluorescence. We discuss different approaches and pitfalls of image registration and its potential combination with Gaussian filtering. This finally allows us to proceed with the investigation of point-by-point correlations.
\end{abstract}

Index Terms-Image registration, CIGS solar cells, X-ray microscopy, X-ray fluorescence (XRF), X-ray beam induced current (XBIC), Gaussian filter

\section{INTRODUCTION}

$\mathrm{X}$-ray microscopy is utilized to characterize solar cells on the nanoscale, profiting from a small X-ray beam size available at third-generation synchrotron beamlines. Due to their high penetration depth, X-rays provide insight into deeper layers of solar cells through X-ray fluorescence (XRF) [1], X-ray excited optical luminescence (XEOL) [2], X-ray diffraction [3], [4], and X-ray beam induced current [5].

While research on mono- and polycrystalline silicon absorbers mainly focuses on metal impurities [6], X-ray microscopy applied to thin-film solar cells allows for the examination of spatial inhomogeneities in performance and elemental distribution that compound-semiconductor absorbers

We acknowledge DESY (Hamburg, Germany), a member of the Helmholtz Association HGF, for the provision of experimental facilities. Parts of this research were carried out at PETRA III. inherently suffer from. The full power unfolds when combining XBIC with further modalities such as XRF [7] to investigate correlations between the charge-collection efficiency and certain elemental concentrations in compound semiconductors like $\mathrm{Cu}(\mathrm{In}, \mathrm{Ga}) \mathrm{Se}_{2}$ (CIGS). For instance, the stoichiometric ratio of gallium to indium has a direct effect on the bandgap of $\mathrm{Cu}(\mathrm{In}, \mathrm{Ga}) \mathrm{Se}_{2}$, and investigating its local variations has led to valuable results in the past [8].

While XBIC and XRF can in principle be measured at the same time [7], this is problematic in the case of X-ray beam induced sample degradation [9]-[12], possibly distorting the XBIC signal while ensuring a significant photon count for fluorescence. The dilemma grows if one desires to measure $\mathrm{X}$-ray diffraction and X-ray excited luminescence, where Xray exposure times need to be increased further to secure a set of data rich in contrast, or if one surveys perovskite solar cells that degrade at a faster rate [10].

Therefore, it may be necessary to perform two or more distinct scans, accepting that there can be drift between the beam and the sample from one scan to the other. The desirable point-by-point correlation of sample properties assessed during distinct scans causes the need for image registration - the process of aligning two images that are slightly shifted to one another.

While the analysis of correlations between nanoscale measurements of the performance and elemental distribution will be the subject of future publications, we focus here on a case study tackling the challenge of obtaining the best spatial alignment of subsequently measured modalities by image registration. 


\section{EXPERIMENTAL}

\section{A. Samples}

The solar cells used for this study were grown at Empa as described in [13] using a $\mathrm{SiO}_{2}$-coated soda-lime glass substrate with a Mo layer as back contact and a multi-stage co-evaporated, low-temperature $\left(<450{ }^{\circ} \mathrm{C}\right)$ CIGS absorber with $\mathrm{NaF}$ and $\mathrm{RbF}$ post-deposition treatment. A chemicalbath deposited $\mathrm{CdS}$ buffer layer, $\mathrm{ZnO}$ window layers, $\mathrm{Ni} / \mathrm{Al}$ grids, and a $\mathrm{MgF}_{2}$ anti-reflective coating complete the cell as represented in Fig. 1.

\section{B. Measurements}

Measurements were conducted at the microprobe endstation of beamline P06 [14], [15] at PETRA III at Deutsches Elektronen-Synchrotron (DESY) in Hamburg, Germany. As shown in Fig. 1, the scans were performed in the plane formed by $Y$ and $Z$, while the $X$ axis corresponds to the $\mathrm{X}$-ray beam axis according to the beamline's coordinate system.

The photon energy was $15.25 \mathrm{keV}$ while the X-ray beam was focused with beryllium compound refractive lenses (CRL) [16] and a correcting phase plate [17] to $105 \mathrm{~nm} \times 108 \mathrm{~nm}$ (vertical $\times$ horizontal, FWHM). Every scan covered an area of $10 \mu \mathrm{m} \times 10 \mu \mathrm{m}$ with a step size of $50 \mathrm{~nm}$. Combining a short dwell time with oversampling has proven to be a reasonable way to increase fluorescence photon counts per unit area in a fly-scan, possibly raising the need for Gaussian filtering.

1) X-ray Beam Induced Current: The wiring for XBIC measurements followed [7] with the front contact grounded to avoid contributions of the replacement current for ejected electrons to the XBIC signal. For maximum signal-to-noise ratio, lock-in amplification [18] was utilized as described in [19]. A chopper modulated the incident X-ray beam at $8.015 \mathrm{kHz}$, and the XBIC signal was demodulated to the chopper frequency using a UHFLI lock-in amplifier from Zurich Instruments after the signal underwent amplification by $1 \mu \mathrm{A} / \mathrm{V}$ through a Stanford Research Systems current preamplifier SR570. The low-pass filter cutoff frequency was set to $501.1 \mathrm{~Hz}$ ( $8^{\text {th }}$ order). For quantification, the XBIC signal was normalized to the photon flux and to the pixel dwell time.

2) X-Ray Fluorescence: X-ray fluorescence photons were detected by a SII Vortex EM silicon drift detector that was coupled to an Xspress3 (Quantum Detectors) read-out system and positioned in close proximity to the sample. Fluorescence spectra were fitted using PyMca as detailed in [20]. The XRF count rate was normalized analogously to XBIC.

3) Scanning: We performed so-called snake-scans in continuous or fly-scan mode where the horizontal direction of the stage was reversed every other line. It is characteristic of a flyscan that dialed stage positions deviate from measured encoder positions, which is why these do not constitute a perfectly rectangular matrix of pixels. Therefore, we interpolated each dataset of $200 \mathrm{px} \times 200 \mathrm{px}$ to a supergrid of $1000 \mathrm{px} \times 1000 \mathrm{px}$ using the nearest-neighbor method. This process is demonstrated for an exemplary XBIC scan in Fig. 2. While Fig. 2(a) depicts the XBIC measurement at the dialed stage positions

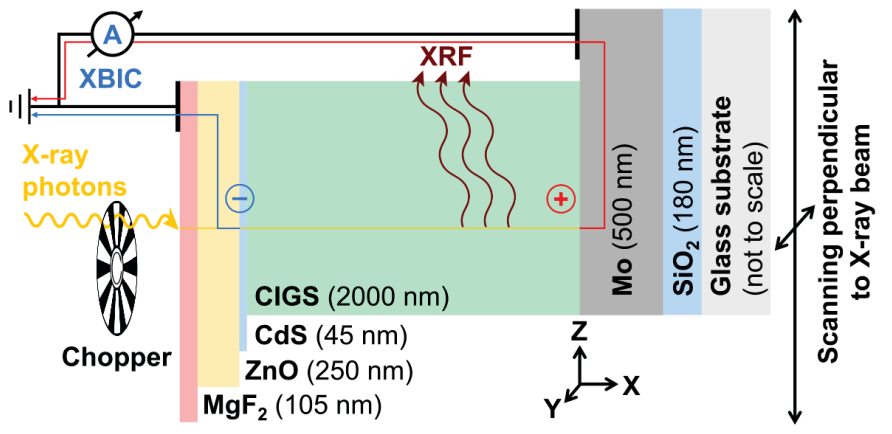

Fig. 1. Schematic setup showing a modulated synchrotron beam inducing a current in the CIGS solar cell. Simultaneously, X-ray fluorescence photons are generated in all layers.

with pixels of $50 \mathrm{~nm} \times 50 \mathrm{~nm}$, Fig. 2(b) represents the XBIC values at the measured stage positions with a supergrid pixel size of $10 \mathrm{~nm} \times 10 \mathrm{~nm}$ to account for deviating stage positions.

4) Dwell Time Settings: During lock-in amplified XBIC measurements, the chopper effectively halves the photon flux, which leads to a decrease in contrast of the XRF signal. As shown in [21], X-ray beam induced sample degradation predominantly affects XBIC, whereas the XRF signal barely changes. Due to the trade-off between an increase in dwell time and possible degradation of the CIGS solar cell, we performed two distinct scans optimized for maximum sensitivity in the XBIC and XRF measurements, respectively:

1) A fast scan was performed using a pixel dwell time of $0.01 \mathrm{~s}$ with the chopper placed in the beam to extract the lock-in amplified XBIC signal. While this scan was optimized for XBIC, XRF was also measured, though with low contrast due to (a) halved X-ray flux by the chopper and (b) short dwell time.

2) A slow scan was performed after the fast scan for optimum XRF signal with a tenfold increase in the dwell time $(0.1 \mathrm{~s})$. The chopper was placed out of the beam, but we still recorded the pre-amplified XBIC signal that we will refer to as the direct signal.
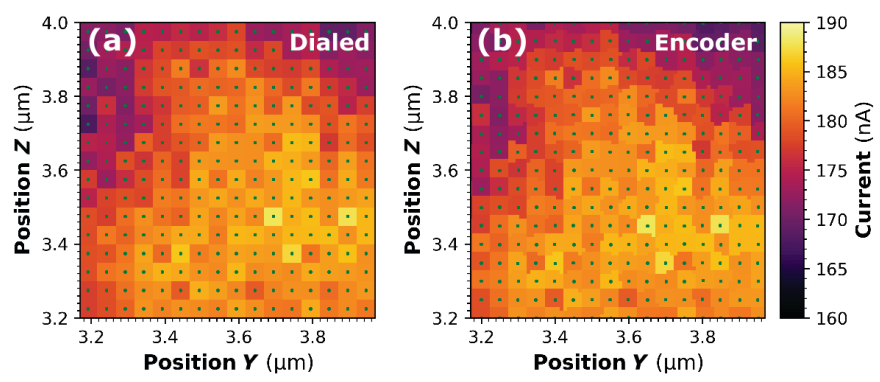

Fig. 2. Cutout of a larger X-ray beam induced current measurement: while (a) shows the measured XBIC at the dialed stage positions with $50 \mathrm{~nm} \times 50 \mathrm{~nm}$ step size, (b) shows the same data at the measured stage position, projected to a supergrid by nearest-neighbor interpolation with $10 \mathrm{~nm} \times 10 \mathrm{~nm}$ pixel size. The green markers indicate the dialed and measured encoder positions, respectively. 


\section{Image Registration}

As we expected the results from the fast and the slow scan to be misaligned due to relative drift of the sample-beam interaction point, we used image registration to evaluate the spatial offset between the fast and slow scans that covered nominally the same area. Thereby, the Enhanced Correlation Coefficient (ECC) maximization method described in [22] was utilized. It is implemented in OpenCV [23], [24] for Python under the function name findtransformECC. Given two 8-bit images, ${ }^{\text {a }}$ it returns a so-called warp matrix of $2 \times 3$ entries

$$
\left[\begin{array}{lll}
a_{00} & a_{01} & b_{1} \\
a_{10} & a_{11} & b_{2}
\end{array}\right]
$$

that describe the transformation of pixel coordinate $(Y, Z)$ (in our case from the slow scan) to the corresponding pixel coordinate $\left(Y^{\prime}, Z^{\prime}\right)$ (in our case from the fast scan) following

$$
\left(\begin{array}{l}
Y^{\prime} \\
Z^{\prime}
\end{array}\right)=\left[\begin{array}{ll}
a_{00} & a_{01} \\
a_{10} & a_{11}
\end{array}\right] \cdot\left(\begin{array}{l}
Y \\
Z
\end{array}\right)+\left[\begin{array}{l}
b_{1} \\
b_{2}
\end{array}\right] .
$$

The parameters $b_{1}$ and $b_{2}$ (in units of pixels) describe a linear transformation in the direction of $Y$ and $Z$, whereas parameters $a_{00}, a_{01}, a_{10}, a_{11}$ cause the input image to rotate or shear, which results in an affine transformation. If the alignment is limited to translation, these four parameters constitute the identity matrix.

The actual image transformation characterized by a warp matrix as well as the interpolation of the newly assigned coordinates $\left(Y^{\prime}, Z^{\prime}\right)$ with respect to the original pixel positions is done by the OpenCV function warpAffine. Pearson's correlation coefficient $\rho$ is used for quantification of the achieved alignment with

$$
\rho_{A, B}=\frac{1}{N_{Z} N_{Y}-1} \sum_{j=1}^{N_{Z}} \sum_{i=1}^{N_{Y}}\left(\frac{A_{i j}-\mu_{A}}{\sigma_{A}}\right)\left(\frac{B_{i j}-\mu_{B}}{\sigma_{B}}\right) .
$$

$A$ and $B$ are images from the fast and slow scan respectively, with $N_{Y}$ rows and $N_{Z}$ columns, as well as values $A_{i j}$ and $B_{i j}$ at a specific pixel coordinate $(i, j)$. The arithmetic mean and the standard deviation of the image data are denoted $\mu$ and $\sigma$, respectively.

The transformation between the fast and the slow scan can be evaluated from either XBIC or XRF images with resulting warp matrices that deviate from one another. To compare the transformation obtained from XBIC or XRF alignment, we denote

1) $\rho_{\text {XBIC-XBIC }}$ the correlation coefficient obtained from $\mathrm{XBIC}$ data of the fast scan and the registered slow scan, and

2) $\rho_{\mathrm{Se}-\mathrm{Se}}$ the correlation coefficient obtained from selenium data of the fast scan and the registered slow scan, with selenium being the element of greatest XRF sensitivity.

\footnotetext{
${ }^{\text {a }}$ Reducing the image depth to 8 bit, outliers in the XRF data required a $98^{\text {th }}$-percentile cutoff for contrast maximization, but not in the XBIC data.
}

\section{Gaussian Filters}

In OpenCV, Gaussian filters for an image $A$ such as the supergrid are controlled through a specific filter size $K$, an odd number that defines the number of pixels that are considered in either dimension for the calculation of the smoothened image $\widetilde{A}$ at a pixel position $\widetilde{A}_{i j}$ as

$$
\widetilde{A}_{i j}=\sum_{n=-\frac{K-1}{2}}^{\frac{K-1}{2}} \sum_{m=-\frac{K-1}{2}}^{\frac{K-1}{2}} G_{m n} \cdot A_{i+m, j+n} .
$$

The Gaussian kernel matrix $G$ effectively weights the pixels of the original image $A$. It consists of $K^{2}$ entries where $(m, n)=(0,0)$ marks the kernel's center that coincides with the coordinates of pixel $A_{i j}$. Each matrix entry $G_{m n}$ is computed as

$$
G_{m n}=\alpha \cdot \exp \left(\frac{m^{2}+n^{2}}{2 \cdot \sigma^{2}}\right)
$$

with a scale factor $\alpha$ such that

$$
\sum_{n=-\frac{K-1}{2}}^{\frac{K-1}{2}} \sum_{m=-\frac{K-1}{2}}^{\frac{K-1}{2}} G_{m n}=1 .
$$

The Gaussian standard deviation in the OpenCV implementation is directly linked to the filter size through

$$
\sigma=0.3 \cdot((K-1) \cdot 0.5-1)+0.8 \text {. }
$$

In our application, Gaussian filters fulfil two purposes:

1) Applied before image registration, a Gaussian filter of size $K_{\text {reg }}$ helps to separate characteristic features from noise and measurement artifacts for optimum image registration, as will be shown in section III-C.

2) Applied after image registration, a Gaussian filter denoted here $K_{\text {img }}$ can be used to smoothen the images to account for oversampling and the projection of the measurement data to a supergrid described in section II-B3.

\section{RESULTS}

\section{A. Unregistered XBIC and XRF Measurements}

The unregistered XBIC and XRF maps that serve as input for this study are visualized in Fig. 3. With regard to fluorescence, we are considering here the count rate of selenium as it is the most abundant element in the $\mathrm{Cu}(\mathrm{In}, \mathrm{Ga}) \mathrm{Se}_{2}$ layer and provides the highest XRF count rate.

Fig. 3(a\&c) were measured during the fast scan mentioned above with a dwell time of $0.01 \mathrm{~s}$ and the chopper placed in the beam. One can clearly perceive the lack of contrast in the XRF plot shown in Fig. 3(c) compared to Fig. 3(d) from the slow scan, where the dwell time was increased tenfold. Removing the chopper effectively doubled the photon count rate. These maps demonstrate that two distinct measurements optimized for XBIC and XRF allowed to extract high-quality $\mathrm{XBIC}$ and XRF data. 

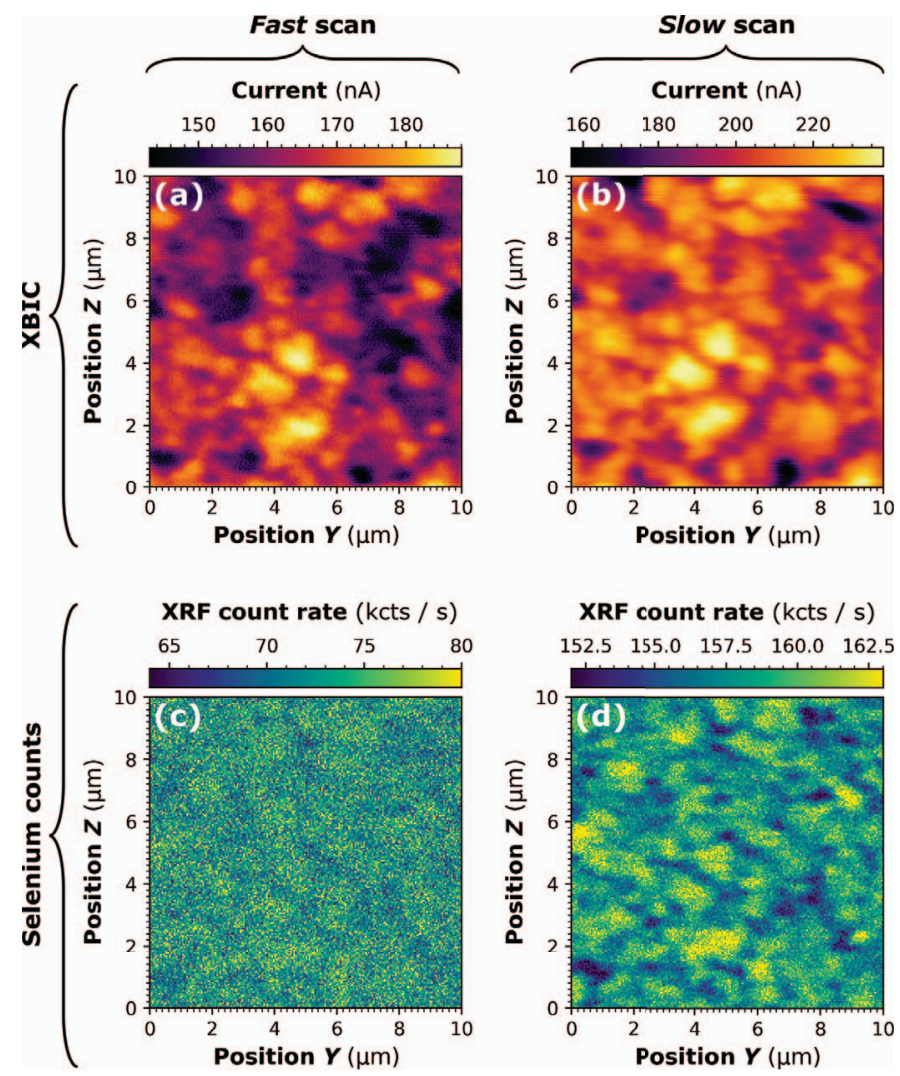

Fig. 3. Multi-modal measurements were conducted in two distinct scans. From the first, fast scan with $0.01 \mathrm{~s}$ pixel dwell time, or effectively $0.005 \mathrm{~s}$ due to the chopper, the lock-in amplified XBIC signal and selenium fluorescence count rate shown in panels $(\mathrm{a} \& \mathrm{c})$ were obtained. From the second, slow scan with $0.1 \mathrm{~s}$ pixel dwell time (measured without chopper), the direct XBIC signal and selenium fluorescence count rate shown in panels (b\&d) were obtained.

While all four images nominally depict the same area of the solar cell, the careful reader will notice an offset when comparing Fig. 3(a\&c) with Fig. 3(b\&d). In addition, we note line artifacts that are predominantly found in the XBIC scan in Fig. 3(b). Here, we suggest two possible sources. First, the XBIC signal suffers from a delay from amplification steps and low-pass filters that lead to an afterglow in the XBIC signal. We can then observe a line mismatch because the scanning direction of the stage reverses every other line. Second, we can observe stage acceleration and deceleration artifacts at the edges of the images caused by the snake-scan (see section II).

\section{B. Preliminary Image Registration}

There are two different datasets at hand that can be used to determine the warp matrix that most accurately describes the shift between the fast and the slow scan:

1) XBIC images from the fast and the slow scan, denoted by (a) and (b) in Fig. 3, and

2) selenium images from the fast and the slow scan, denoted by (c) and (d) in Fig. 3.

Every image is cropped by $100 \mathrm{px}(1 \mu \mathrm{m})$ on the right and on the left to exclude stage acceleration and deceleration artifacts. From this point on, we will only use these cropped images.
The steps described in section II-C were applied, starting without employing a Gaussian filter beforehand. In this way, we obtained two different warp matrices from comparing the XBIC images in Fig. 3(a\&b) as well as the XRF images in Fig. 3(c\&d).

As displayed in the first line of Tab. I, the resulting warp matrices suggest a translation in negative $Y$ and positive $Z$ direction to account for the spatial offset between the fast and the slow scan. However, the matrices deviate from one another by $2.97 \mathrm{px}$ in $Y$ and by $3.49 \mathrm{px}$ in $Z$, resulting in two available alignments for images obtained from the same two scans. The maximum discrepancy of $3.49 \mathrm{px}$ corresponds to $34.9 \mathrm{~nm}$.

Pearson's correlation coefficient is used to assess the achieved alignment. The coefficients $\rho_{\text {XBIC-XBIC }}$ and $\rho_{\text {Se-Se }}$ are displayed in the second and third line of Tab. I. Note that the overall correlation of the aligned XBIC images is higher than that of the corresponding selenium images, which is predominantly caused by the noise apparent in the selenium data from the fast scan (see Fig. 3(c)).

Comparing the correlation coefficients of the alignment computed from the XBIC images (middle column of Tab. I) and from the selenium images (right column of Tab. I), we note that the correlation coefficients from the XBIC images are marginally higher - thus suggesting that this provides the best alignment.

\section{Evaluation of Different Gaussian Filters}

With an X-ray beam size of $105 \mathrm{~nm} \times 108 \mathrm{~nm}$, one can account for oversampling, noise and artifacts by applying a Gaussian filter of $K_{\mathrm{img}}=K_{\text {reg }}=33$ px on the registered images (corresponding to $\sigma_{\text {reg }}=53 \mathrm{~nm}$ ) and therefore resembles the X-ray beam profile. To study the effects of $K_{\text {reg }}$ and $K_{\text {img }}$, we ran the image registration algorithm for both XBIC and selenium datasets, employing different Gaussian filters in the range of 3 px to $49 \mathrm{px}$.

Correlation coefficients were first evaluated as a function of $K_{\text {reg }}$, meaning that warp matrices were computed from progressively smoothed images prior to calculating the correlation coefficients of the aligned images blurred with a constant filter $K_{\text {img }}=33$ px. The result is represented in Fig. 4(a\&c). Red and blue markers indicate whether XBIC or selenium served as underlying dataset for registration. While there are

TABLE I

WARP MATRICES AND PEARSON'S CORRELATION COEFFICIENTS OBTAINED FROM THE REGISTRATION OF IMAGES FROM THE fast AND THE slow SCAN WITHOUT APPLYING A GAUSSIAN FILTER

\begin{tabular}{|c|c|c|c|c|c|}
\hline $\begin{array}{l}\text { Set of data for cal- } \\
\text { culation of warp matrix }\end{array}$ & $\begin{array}{l}X \\
\text { Fig. }\end{array}$ & $\begin{array}{l}\text { IC } \\
(\mathrm{a} \& b)\end{array}$ & & ele & um \\
\hline Warp matrix & {$\left[\begin{array}{ll}1 & 0 \\
0 & 1\end{array}\right.$} & $\left.\begin{array}{l}-4.28 \\
39.29\end{array}\right]$ & & $\begin{array}{l}0 \\
1\end{array}$ & $\left.\begin{array}{l}-1.31 \\
35.80\end{array}\right]$ \\
\hline $\begin{array}{c}\rho_{\mathbf{X B I C}-\mathbf{X B I C}} \\
\text { fast and slow scan }\end{array}$ & \multicolumn{2}{|c|}{0.800} & \multicolumn{3}{|c|}{0.797} \\
\hline $\begin{array}{c}\rho_{\text {Se-Se }} \\
\text { fast and slow scan }\end{array}$ & \multicolumn{2}{|c|}{0.181} & \multicolumn{3}{|c|}{0.178} \\
\hline
\end{tabular}



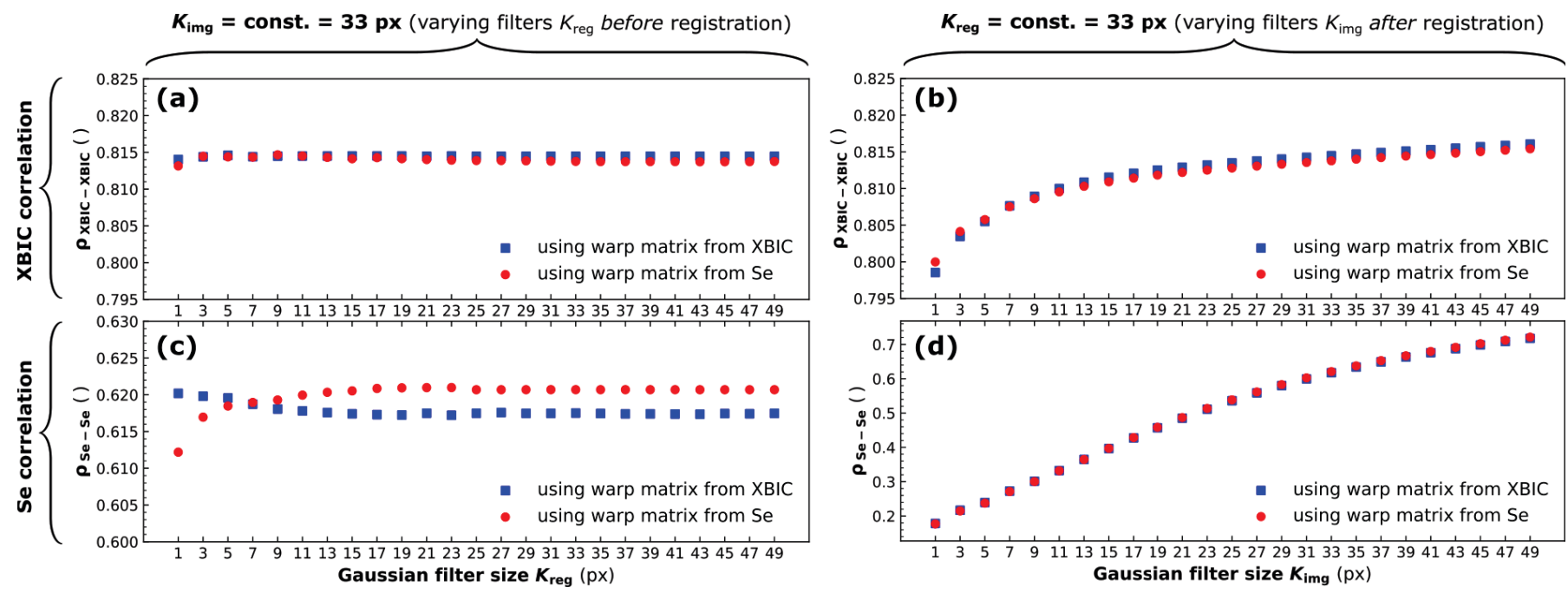

Fig. 4. Pearson's correlation coefficients as in Tab. I are first used in (a) and (c) to evaluate the effect of Gaussian filters $K_{\text {reg }}$ on the computed warp matrices and thus the alignment achieved through image registration employing the XBIC signal or the selenium count rate from the fast and slow scan. Here, the Gaussian filter $K_{\text {img }}$ applied after image registration is maintained constant at $33 \mathrm{px}\left(\sigma_{\text {img }}=53 \mathrm{~nm}\right)$, which is motivated by the X-ray beam size $(\sim 106 \mathrm{~nm}$ FWHM). In (b) and (d), we study the effect of varying filters $K_{\text {img }}$ on the resulting correlation coefficients with constant warp matrices obtained with a filter size $K_{\text {reg }}=33 \mathrm{px}$. In general, a filter size of $1 \mathrm{px}$ means that no Gaussian filter was applied.

minor fluctuations in the correlation coefficients for filter sizes $K_{\text {reg }} \leq 9 \mathrm{px}$, both XBIC and selenium image couples appear to provide a robust alignment for $K_{\text {reg }}>9$ px. The curves do neither strongly depend on the registering modality nor a precise Gaussian filter size when the alignment is consistently quantified with images blurred with a filter size $K_{\text {img }}=33 \mathrm{px}$.

Having found this, correlation coefficients were secondly evaluated as a function of $K_{\mathrm{img}}$ while maintaining alignment and warp matrices from XBIC and selenium count rate constant, calculated at $K_{\text {reg }}=33$ px. Both Fig. 4(b\&d) unveil an increase in the correlation coefficients with the Gaussian filter size $K_{\text {img }}$. This increase is minor for $\rho_{\text {XBIC-XBIC }}$ and can be attributed in part to smoothing of line artifacts and noise in the XBIC scans in Fig. 3(a\&b). In contrast, the Gaussian filter causes $\rho_{\mathrm{Se}-\mathrm{Se}}$ to more than triple over a range of $K_{\text {reg }}=1$ to $K_{\text {reg }}=49$, which can be attributed to the efficient suppression of high-frequency noise in the low-contrast selenium image from the fast scan (Fig. 3(c). Note that Gaussian filters $K_{\text {img }}$ that are much larger than the X-ray beam profile imply that apart from noise, features of interest could be washed out. Again, warp matrices computed from XBIC and Selenium datasets eventuate in similar correlation coefficients.

Based on these considerations, we choose to apply a Gaussian filter size of $K_{\text {reg }}=33$ px before image registration. In an effort to account for oversampling and reducing noise without sacrificing resolution, we also choose a filter size of $K_{\text {img }}=33 \mathrm{px}$ to be applied after registration. Due to fewer systematic measurement artifacts of XRF compared to XBIC measurements, we further consider the warp matrix from $\mathrm{Se}-$ Se for optimum image registration.

A different approach to visualize the process of image registration is shown in Fig. 5. Based on the selenium XRF count rate shown in Fig. 3, we study the impact of systematically varied warp matrices on the correlation coefficient $\rho_{\mathrm{Se}-\mathrm{Se}}$ for (a) no Gaussian filter and (b) a Gaussian filter of size $K_{\text {img }}=$ $33 \mathrm{px}$. The axes correspond to the parameters $b_{1}$ and $b_{2}$ that were introduced in (1) and describe the horizontal and vertical translation in the warp matrices. The alignment identified to be optimal before (Se-Se, $K_{\text {reg }}=K_{\text {img }}=33 \mathrm{px}$ ) is indicated by a white cross. One can clearly see irregularities in Fig. 5(a) due to noise dominant in the fast XRF scan (see Fig. 3(c)). On the contrary, Fig. 5(b) appears smoother, presenting a more forgiving environment for the ECC maximization algorithm.

\section{Optimized Image Registration with Translation}

Similar to the results shown in Tab. I, warp matrices computed from XBIC-XBIC and Se-Se comparison utilizing
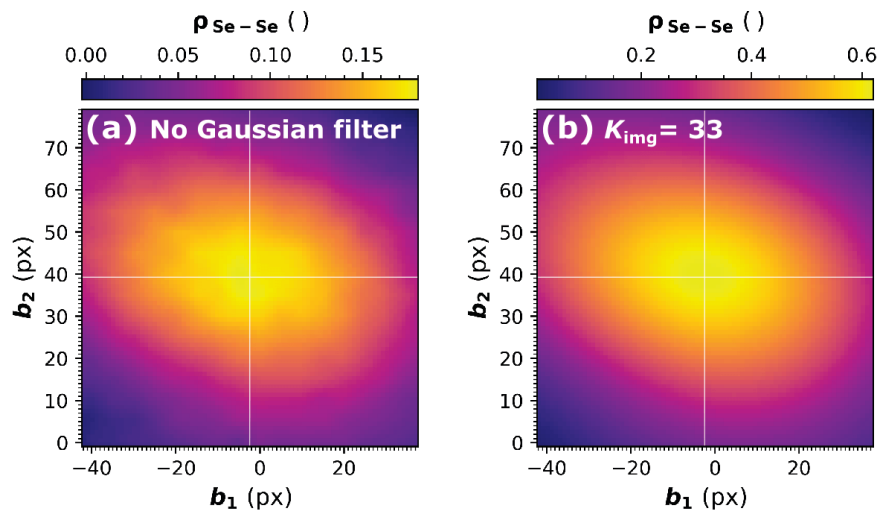

Fig. 5. The correlation coefficient $\rho_{\mathrm{Se}-\mathrm{Se}}$ is visualized for varying alignments described by parameters $b_{1}$ and $b_{2}$ in the warp matrices. In panel (a), no Gaussian filter was applied, whereas a Gaussian filter with $K_{\text {img }}=33 \mathrm{px}$ was applied to the images in panel (b). The white cross marks the alignment found with a Gaussian filter size $K_{\mathrm{reg}}=K_{\mathrm{img}}=33 \mathrm{px}$. 
TABLE II

OPTIMIZED WARP MATRICES AND PEARSON'S CORRELATION COEFFICIENTS OBTAINED FROM THE REGISTRATION OF IMAGES FROM THE fast AND THE slow SCAN WITH A GAUSSIAN FILTER OF SIZE $K=33 \mathrm{px}$

\begin{tabular}{ccc}
\hline $\begin{array}{c}\text { Set of data for cal- } \\
\text { culation of warp matrix }\end{array}$ & $\begin{array}{c}\text { XBIC } \\
\text { Fig. 3(a\&b) }\end{array}$ & $\begin{array}{c}\text { Selenium } \\
\text { Fig. 3(c\&d) }\end{array}$ \\
\hline Warp matrix & {$\left[\begin{array}{ccc}1 & 0 & -3.11 \\
0 & 1 & 37.28\end{array}\right]$} & {$\left[\begin{array}{lll}1 & 0 & -1.90 \\
0 & 1 & 39.49\end{array}\right]$} \\
\hline $\begin{array}{c}\boldsymbol{\rho}_{\mathbf{X B I C}-\mathbf{X B I C}} \\
\text { fast } \text { and slow scan } \\
\begin{array}{c}\boldsymbol{\rho}_{\text {Se-Se }} \\
\text { fast } \text { and slow scan }\end{array}\end{array}$ & 0.814 & 0.814 \\
\hline
\end{tabular}

now a Gaussian filter of size $K_{\text {reg }}=33 \mathrm{px}$ are displayed in Tab. II. Also, the correlation coefficients $\rho_{\text {XBIC-XBIC }}$ and $\rho_{\text {Se-Se }}$ can be read from Fig. 4 for filter size $K_{\text {reg }}=K_{\text {img }}=33$ px and are additionally listed in Tab. II for the sake of comparability with Tab. I. The correlation has increased due to (a) better alignment of the images and (b) smoothing by the Gaussian filter.

Fig. 6(a\&b) demonstrate the effect of the Gaussian filter with size $K_{\text {reg }}=33 \mathrm{px}$ on the selenium images from the fast and the slow scan after their conversion to 8-bit greyscale as required by the function findtrans formECC. Fig. 6(c\&d) illustrate the resulting alignment of the high-sensitivity selenium count rate from the slow scan (Fig. 3(d)) with the high-sensitivity XBIC signal from the fast scan (Fig. 3(a)). Fig. 6(c) results directly from the fast XBIC measurement shown Fig. 3(a) after cropping left and right, and applying a Gaussian filter of size $K_{\mathrm{img}}=33 \mathrm{px}$ that matches the beam size. For spatial correlation to this image, the XRF signal from the slow measurement shown in Fig. 3(d) was registered to the image Fig. 6(c) employing Fig. 6(a\&b). Compared to the preliminary results in Fig. 3, the offset between both images is minimized in the aligned and smoothened images in Fig. 6(c\&d). The consideration of all sample coordinates that are covered in both scans will allow for a point-by-point correlation of the XBIC signal and elemental distribution that is, however, beyond the scope of this study.

\section{E. Image Registration with an Affine Transformation}

If the probe-sample drift is not limited to two-dimensional translations in directions of $Y$ and $Z$ but involves an angular component, entries $a_{00}, a_{01}, a_{10}$ and $a_{11}$ of the corresponding warp matrix need to describe rotation and stretching of the image, which is called an affine transformation in OpenCV.

Such a case is shown in Fig. 7(a\&b), where the XBIC measurements appear clinched and stretched, respectively. This may have been caused e.g. by external stress applied to the solar cell through the wiring due to improper stress relief, causing it to tilt. The findtransformECC algorithm can account for this by utilizing the affine warp mode.

To obtain an initial guess of the underlying affine transformation, we compare the scans that are to be aligned and manually map features that appear to belong to the same spot
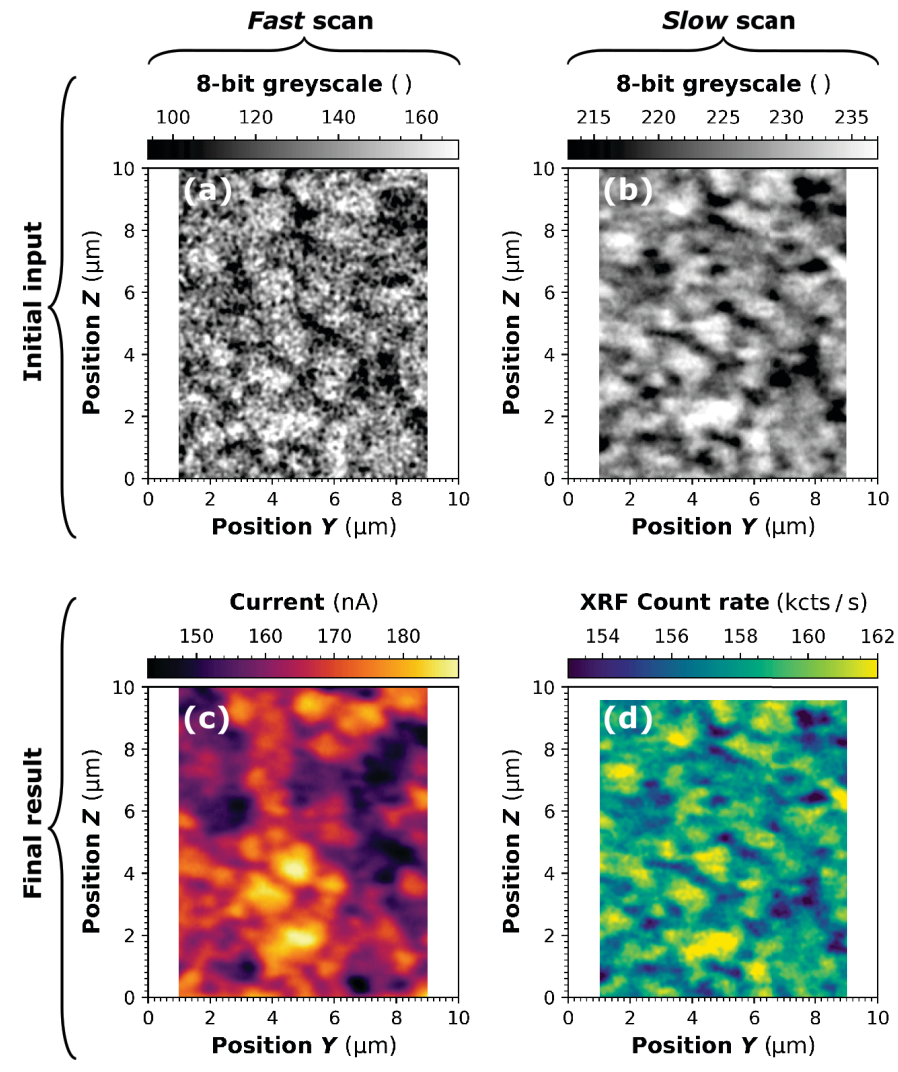

Fig. 6. Overview of the optimized image registration: (a) and (b) depict the selenium distribution from the fast and the slow scan as taken by the algorithm: both were converted to 8-bit greyscale, cropped by $100 \mathrm{px}$ on the left and on the right and blurred with a Gaussian filter of size $K_{\text {reg }}=33$ px. (c) and (d) depict the final result: XBIC and selenium images, both smoothened with a Gaussian filter of size $K_{\mathrm{img}}=33 \mathrm{px}$, after (d) has been aligned with XBIC from (c) using the warp matrix obtained from (a) and (b).

on the cell. This process is visualized by colored markers in Fig. 7(a-d). These lists of coordinates are passed to function estimateAffine2D; the returned warp matrix applied to Fig. 7(b) results in the first manually aligned image shown in Fig. 7(c). This estimate is then passed as starting values to the actual ECC maximization algorithm. The final alignment is represented in the affine warp matrix

$$
\left[\begin{array}{ccc}
0.982 & -0.057 & -1.31 \\
0.003 & 1.11 & 68.450
\end{array}\right]
$$

and its effect is shown in Fig. 7(d).

\section{Discussion}

We have performed image registration under consideration of different Gaussian filters and two distinct registering modalities, namely XBIC and selenium data. While they nominally describe the same offset, the various warp matrices found in section III differ by a maximum of $3.49 \mathrm{px}$ in the supergrid, which corresponds to $34.9 \mathrm{~nm}$ and is below the resolution of the experiment. Based on the small variations of the warp matrices and the resulting correlation coefficients, we conclude that the obtained alignment appears to be robust. 

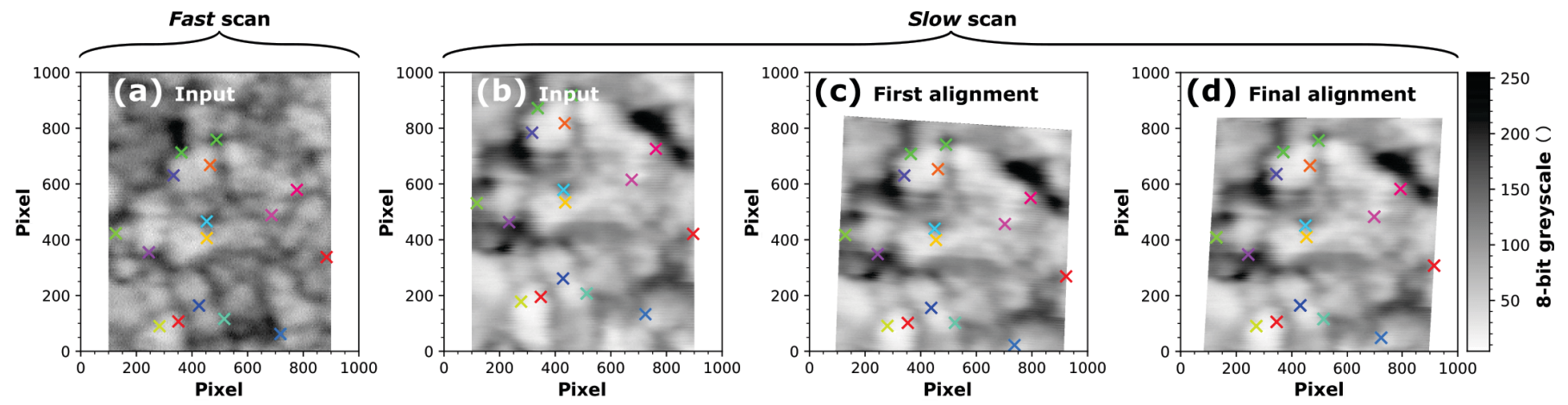

Fig. 7. Steps in the alignment process of two scans with multi-dimensional distortion relative to each other. Subfigures (a) and (b) show the cropped XBIC input images converted to 8-bit greyscale. Image registration in the same way as Fig. 6(a\&b) constrained to translation failed. Instead, an affine transformation is required, which was initialized by manual feature mapping (see colored markers). The initial alignment (c) then enabled the algorithm to converge in (d).

The actual research goal - examining the impact of elemental concentrations in the CIGS layer of the solar cell on the cell performance - will require the calculation of correlation coefficients from XBIC and fluorescence data. This is why we compare in Tab. III the correlation coefficient $\rho_{\mathrm{XBIC}-\mathrm{Se}}$ between the XBIC signal from Fig. 3(a) and the selenium count rate from Fig. 3(d) for all four warp matrices in Tab. I and Tab. II.

The comparison of the correlation coefficient values $\rho_{\text {XBIC-Se }}$ unveil two distinct groups: $0.410 \leq \rho_{\text {XBIC-Se }} \leq 0.415$ without the application of a Gaussian filter after registration, but $0.496 \leq \rho_{\text {XBIC-Se }} \leq 0.500$ with a Gaussian filter of size $K_{\text {img }}=33 \mathrm{px}$. This means first that a small difference in image alignment below the measurement resolution only has a minor impact, and second that the application of a Gaussian filter leads to a non-negligible increase of the correlation coefficient. While the first observation reconfirms the robustness of our image registration approach, the second observation indicates that by reducing the effect of high-frequency noise, Gaussian filters can significantly impact the absolute correlation coefficient such that utmost care is required in quantitative analyses.

As an example for future analyses, Fig. 8 visualizes the correlation of XBIC (fast scan) and selenium count rate (slow scan) based on the image registration and Gaussian filter settings as detailed in the last line in Tab. III. The XBIC

TABLE III

CORRELATION OF XBIC AND THE REGISTERED SELENIUM IMAGE UTILIZING DIFFERENT WARP MATRICES AND OPTIONALLY APPLYING A GAUSSIAN FILTER AFTER REGISTRATION

\begin{tabular}{cccc}
$\begin{array}{c}\text { Gaussian filter } \\
\text { before registration }\end{array}$ & Warp matrix & $\begin{array}{c}\text { Gaussian filter } \\
\text { after registration }\end{array}$ & $\rho_{\mathbf{X B I C - S e}}$ \\
\hline- & {$\left[\begin{array}{lll}1 & 0 & -4.28 \\
0 & 1 & 39.29\end{array}\right]$} & $K_{\mathrm{img}}=33 \mathrm{px}$ & 0.415 \\
- & {$\left[\begin{array}{lll}1 & 0 & -1.31 \\
0 & 1 & 35.80\end{array}\right]$} & $K_{\mathrm{img}}=33 \mathrm{px}$ & 0.500 \\
\hline \multirow{2}{*}{$K_{\mathrm{reg}}=33 \mathrm{px}$} & {$\left[\begin{array}{lll}1 & 0 & -3.11 \\
0 & 1 & 37.28\end{array}\right]$} & $K_{\mathrm{img}}=33 \mathrm{px}$ & 0.410 \\
\hline$K_{\mathrm{reg}}=33 \mathrm{px}$ & {$\left[\begin{array}{lll}1 & 0 & -1.90 \\
0 & 1 & 39.49\end{array}\right]$} & $K_{\mathrm{img}}=33 \mathrm{px}$ & 0.499 \\
\hline
\end{tabular}

image was registered with the transformation obtained from the selenium dataset with a Gaussian filter of size $K_{\text {reg }}=33 \mathrm{px}$ to extract a warp matrix, as shown in Tab. II. Afterwards, a Gaussian filter of the same size $K_{\mathrm{img}}=33 \mathrm{px}$ was applied to minimize the effects of noise and artifacts.

\section{CONCLUSions}

We have demonstrated how to effectively register images from two distinct scanning X-ray microscopy measurements. Herein, we have utilized the ECC maximization algorithm under consideration of a Gaussian filter that matches the $\mathrm{X}$-ray beam profile to minimize the impact of noise while maintaining characteristic features of the solar cell. Several aspects have been useful for optimizing the image registration:

- Selecting regions of interest to reduce the impact of known artifacts and distortions,

- Choosing the optimal Gaussian-filter size both prior to registration and after registration for data correlation,

- Deploying sophisticated alignment methods such as an affine transformation if necessary.

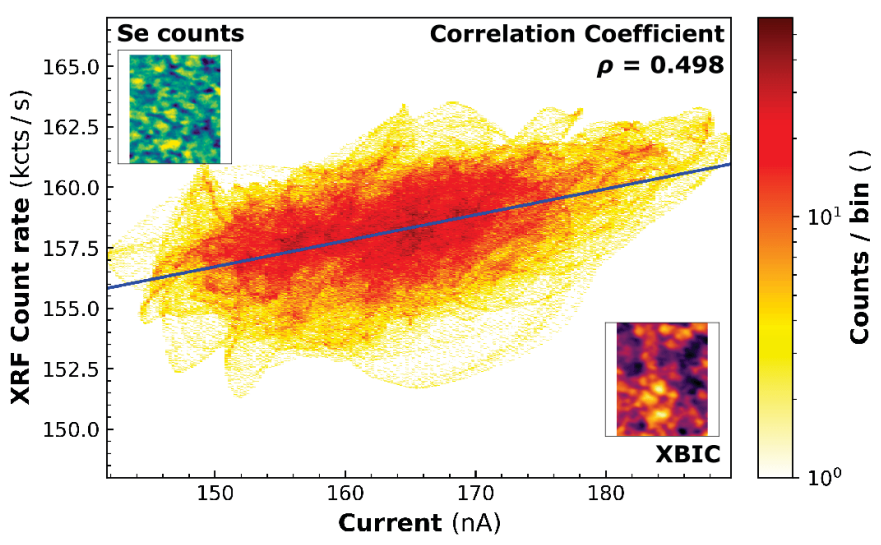

Fig. 8. Correlation of XBIC and selenium count rate from XRF after registration with $K_{\mathrm{reg}}=33 \mathrm{px}$ and subsequent application of a Gaussian filter $K_{\text {img }}=33 \mathrm{px}$ to both images visualized in a two-dimensional histogram. Every pixel bins supergrid data points visible in both images and is colored logarithmically according to the number of data points in a pixel. The insets correspond to Fig. 6(c\&d). The line is the result of a linear fit. 
Image registration may be even more relevant in future scanning X-ray microscopy measurements: $4^{\text {th }}$-generation synchrotrons such as the planned PETRA IV [25] will feature a further increase in X-ray brilliance, providing better conditions for photon-hungry techniques such as X-ray excited optical luminescence (XEOL). Multi-modal measurements including XEOL, XRF and XBIC may then need to be performed consecutively due to incompatible measurement conditions or different setups, and image registration is going to be vital especially when it comes to degrading samples.

Nevertheless, it should be kept in mind that image registration will always be the second choice only for the correlation of data from different measurement modalities. The most confident correlation is obtained from multi-modal measurements with the simultaneous assessment of the parameters of interest.

\section{ACKNOWLEDGMENT}

We greatly acknowledge Torsten Boese, Stephan Botta, Dennis Brückner, Ralph Döhrmann, Johannes Hagemann, Christian G. Schroer, Frank Seiboth, Martin Seyrich, Kathryn Spiers, Patrik Wiljes (Deutsches Elektronen-Synchrotron, Germany), Shiro Nishiwaki, Ayodhya N. Tiwari, Romain Carron, Enrico Avancini (Empa, Switzerland), Jan Flügge, Tobias Kipp, Andreas Kolditz, Alf Mews, Jan Siebels, and Christian Strelow (Universität Hamburg, Germany) for technical support, providing solar cells, and fruitful discussions.

\section{REFERENCES}

[1] B. West, M. Stuckelberger, A. Jeffries, S. Gangam, B. Lai, B. Stripe, J. Maser, V. Rose, S. Vogt, and M. I. Bertoni, "X-ray fluorescence at nanoscale resolution for multicomponent layered structures: a solar cell case study," Journal of Synchrotron Radiation, vol. 24, pp. 288-295, 2017.

[2] R. Taylor, A. Finch, J. Mosselmans, and P. Quinn, "The development of a XEOL and TR XEOL detection system for the I18 microfocus beamline Diamond light source," Journal of Luminescence, vol. 134, pp. 49-58, 2013.

[3] A. Ulvestad, S. O. Hruszkewycz, M. V. Holt, M. O. Hill, I. CalvoAlmazán, S. Maddali, X. Huang, H. Yan, E. Nazaretski, Y. S. Chu, L. J. Lauhon, N. Rodkey, M. I. Bertoni, and M. E. Stuckelberger, "Multimodal x-ray imaging of grain-level properties and performance in a polycrystalline solar cell," Journal of Synchrotron Radiation, vol. 26 , pp. 1316-1321, Jul 2019.

[4] I. Calvo-Almazán, A. P. Ulvestad, E. Colegrove, T. Ablekim, M. V. Holt, M. O. Hill, S. Maddali, L. J. Lauhon, M. I. Bertoni, X. Huang, H. Yan, E. Nazaretski, Y. S. Chu, S. O. Hruszkewycz, and M. E. Stuckelberger, "Strain mapping of CdTe grains in photovoltaic devices," IEEE Journal of Photovoltaics, vol. 9, no. 6, pp. 1790-1799, 2019.

[5] O. F. Vyvenko, T. Buonassisi, A. A. Istratov, H. Hieslmair, A. C. Thompson, R. Schindler, and E. R. Weber, "X-ray beam induced current-a synchrotron radiation based technique for the in situ analysis of recombination properties and chemical nature of metal clusters in silicon," Journal of Applied Physics, vol. 91, no. 6, pp. 3614-3617, 2002.

[6] T. Buonassisi, A. A. Istratov, M. D. Pickett, M. A. Marcus, G. Hahn, R. S., J. Isenberg, W. Warta, G. Willeke, T. F. Ciszek, and E. R. Weber, "Quantifying the effect of metal-rich precipitates on minority carrier diffusion length in multicrystalline silicon using synchrotronbased spectrally resolved x-ray beam-induced current," Applied Physics Letters, vol. 87, no. 4, p. 044101, 2005.

[7] M. Stuckelberger, B. West, T. Nietzold, B. Lai, J. M. Maser, V. Rose, and M. I. Bertoni, "Review: Engineering solar cells based on correlative X-ray microscopy," Journal of Materials Research, vol. 32, no. 10, pp. 1825-1854, 2017
[8] B. West, M. Stuckelberger, H. Guthrey, L. Chen, B. Lai, J. Maser, V. Rose, W. Shafarman, M. Al-Jassim, and M. I. Bertoni, "Grain engineering: How nanoscale inhomogeneities can control charge collection in solar cells," Nano Energy, vol. 32, pp. 488-493, 2017.

[9] S. M. Polvino, C. E. Murray, Ö. Kalenci, I. Noyan, B. Lai, and Z. Cai, "Synchrotron microbeam X-ray radiation damage in semiconductor layers," Applied Physics Letters, vol. 92, no. 22, p. 224105, 2008.

[10] M. Stuckelberger, T. Nietzold, G. N. Hall, B. West, J. Werner, B. Niesen, C. Ballif, V. Rose, D. P. Fenning, and M. I. Bertoni, "Charge collection in hybrid perovskite solar cells: relation to the nanoscale elemental distribution," IEEE Journal of Photovoltaics, vol. 7, no. 2, pp. 590-597, 2017.

[11] M. Stuckelberger, T. Nietzold, B. M. West, R. Farshchi, D. Poplavskyy, J. Bailey, B. Lai, J. Maser, and M. I. Bertoni, "How does CIGS performance depend on temperature at the microscale?," IEEE Journal of Photovoltaics, vol. 8, pp. 278-287, Jan 2018.

[12] M. E. Stuckelberger, T. Nietzold, B. M. West, Y. Luo, X. Li, J. Werner, B. Niesen, C. Ballif, V. Rose, D. P. Fenning, and M. I. Bertoni, "Effects of X-rays on perovskite solar cells," submitted, 2020.

[13] E. Avancini, R. Carron, B. Bissig, P. Reinhard, R. Menozzi, G. Sozzi, S. Di Napoli, T. Feurer, S. Nishiwaki, S. Buecheler, et al., "Impact of compositional grading and overall $\mathrm{Cu}$ deficiency on the near-infrared response in $\mathrm{Cu}$ (In, Ga) Se2 solar cells," Progress in Photovoltaics: Research and Applications, vol. 25, no. 3, pp. 233-241, 2017.

[14] C. G. Schroer, P. Boye, J. M. Feldkamp, J. Patommel, D. Samberg, A. Schropp, A. Schwab, S. Stephan, G. Falkenberg, G. Wellenreuther, and N. Reimers, "Hard X-ray nanoprobe at beamline P06 at PETRA III," Nuclear Instruments and Methods in Physics Research A, vol. 616, pp. 93-96, 2010.

[15] A. Schropp, R. Döhrmann, S. Botta, D. Brückner, M. Kahnt, M. Lyubomirskiy, C. Ossig, M. Scholz, M. Seyrich, P. Wiljes, F. Wittwer, M. E. Stuckelberger, J. Garrevoet, G. Falkenberg, Y. Fam, T. L. Sheppard, J.-D. Grunwaldt, and C. G. Schroer, "PtyNAMi: Ptychographic Nano-Analytical Microscope," Journal of Applied Crystallography, 2020. (in press).

[16] B. Lengeler, C. G. Schroer, M. Richwin, J. Tümmler, M. Drakopoulos, A. Snigirev, and I. Snigireva, "A microscope for hard x rays based on parabolic compound refractive lenses," Applied Physics Letters, vol. 74, no. 26, pp. 3924-3926, 1999.

[17] F. Seiboth, A. Schropp, M. Scholz, F. Wittwer, C. Rödel, M. Wünsche, T. Ullsperger, S. Nolte, J. Rahomäki, K. Parfeniukas, S. Giakoumidis, U. Vogt, U. Wagner, C. Rau, U. Boesenberg, J. Garrevoet, G. Falkenberg, E. C. Galtier, H. J. Lee, B. Nagler, and C. G. Schroer, "Perfect X-ray focusing via fitting corrective glasses to aberrated optics," Nat. Comm., vol. 8, p. 14623, 2017.

[18] M. L. Meade, Lock-in amplifiers: principles and applications. Peter Peregrinus Ltd., 1983.

[19] C. Ossig, T. Nietzold, B. M. West, M. I. Bertoni, G. Falkenberg, C. G. Schroer, and M. E. Stuckelberger, "X-ray beam induced current measurements for multi-modal x-ray microscopy of solar cells," Journal of Visual Experiments, p. e60001, 2019.

[20] C. Ziska, C. Ossig, N. Pyrlik, R. Carron, E. Avancini, G. Fevola, A. Kolditz, J. Siebels, T. Kipp, Z. Cai, M. V. Holt, and M. E. Stuckelberger, "Quantifying the elemental distribution in solar cells from $\mathrm{X}$-ray fluorescence measurements with multiple detector modules," Proc. Photovoltaic Specialist Conference (PVSC), 2020.

[21] M. Kodur, R. E. Kumar, Y. Luo, D. N. Cakan, X. Li, M. E. Stuckelberger, and D. P. Fenning, "X-ray microscopy of halide perovskites: Techniques, applications, and prospects," Advanced Energy Materials, p. 1903170, 2020.

[22] G. D. Evangelidis and E. Z. Psarakis, "Parametric image alignment using enhanced correlation coefficient maximization," IEEE Computer Society, vol. $30,2008$.

[23] OpenCV, "Open source computer vision library," 2015. Available at: https://opencv.org, (accessed June 19, 2020).

[24] G. Bradski, “The OpenCV Library," Dr. Dobb's Journal of Software Tools, 2000

[25] C. G. Schroer, I. Agapov, W. Brefeld, R. Brinkmann, Y.-C. Chae, H.-C. Chao, M. Eriksson, J. Keil, X. Nuel Gavaldà, R. Röhlsberger, O. H. Seeck, M. Sprung, M. Tischer, R. Wanzenberg, and E. Weckert, "PETRA IV: the ultralow-emittance source project at DESY," Journal of Synchrotron Radiation, vol. 25, no. 5, pp. 1277-1290, 2018. 